\title{
Cherry Trees Crown Formation Following the System „Natural Crown Improvement with Reduced Volume”
}

\author{
Valerian BALAN ${ }^{1 *}$, Igor IVANOV ${ }^{1}$ \\ ${ }^{1}$ Faculty of Horticulture, Agrarian State University of Moldova. \\ *)corresponding author, e-mail: v.balan@uasm.md \\ BulletinUASVM Horticulture 72(2) / 2015 \\ Print ISSN 1843-5254, Electronic ISSN 1843-5394 \\ DOI:10.15835/buasvmcn-hort:11341
}

\begin{abstract}
In the present paper we studied the cherry trees crown formation tracking natural improved low volume crown system. The orchard was established in 2010 with 'Ferrovia', 'Adriana' and 'Skeena' cultivars grafted on 'Gisela 6' rootstock (Prunus cerasus x Prunus canescens), at a planting distance of 4 × $2 \mathrm{~m}$. The trees grow up to 2.5-3 $\mathrm{m}$ height, with a trunk of 70-80 cm, then 2 levels of scaffold branches are inserted at intervals of 80-90 cm. In the first level, 3 to 4 branches are arranged uniformly around the axis in spiral at a distance of 8-12 cm between them. Three-sections extend at a height of $80-90 \mathrm{~cm}$ from the top or at $150-180 \mathrm{~cm}$ from the ground fulfil the medium part of the crown, and the third level is represented only by the garnishing branches.
\end{abstract}

Keywords: branches, cultivar, shoots, sweet cherry, training system.

\section{INTRODUCTION}

Extensive crown shapes that require the formation of solid skeletal elements are progressively replaced by flattened or fusiform crown systems in orchards. Systems of low volume crown associated with small planting distances are essential in order to ensure the efficient use of solar energy, fruit production, high labor productivity (cutting, harvesting) etc.

This consideration relies by the research approach of a wide range of crown forms. For instance, the low vigor cultivar/rootstock combinations are indicated in the low volume crown systems as follows: Super Slender Axe (SSA) Upright fruiting offshoots (UFO), Tall Spindle Shafts (TSA), Kym Green Bush (KGB) (Lynn E. Long et al. 2014; Asanica et al. 2013). These crown shapes are indicated for precocious and high yields cultivars looking forward to a simplification method for crown formation by pruning (Balan, 2009).

Practical rational and perfected use of low volume wreath forms to obtain competitive world market fruit production, labor efficiency becomes an issue of great value to modern cherry orchards.

\section{MATERIALS AND METHODS}

The experiment was carried out in a private orchard in Republic of Moldova center, Negureni village. The orchard was established in 2010 with 'Ferrovia', 'Adriana' and 'Skeena' cultivars grafted on 'Gisela 6' rootstock (Prunus cerasus x Prunus canescens), at a planting distance of $4 \times 2 \mathrm{~m}$.

To achieve the proposed goals, principles were developed for crown formation and for branch pruning using the "natural crown improved low volume" system. Currently the orchard is in good condition, maintenance of plantation was performed at optimal times. Crown formation was conducted to improve the balance between vegetative growth and reproductive organs in order to speed up the appearance of fruit on trees.

The experience includes 4 repetitions of 8 trees each. The measurements were performed in laboratory and field conditions in accordance with the approved research methods described 
by Balan et al. (2001). The number, average length and sum of annual growths have been effectuated at 32 trees in variant.

\section{RESULTS AND DISCUSSION}

During the vegetation of 2010, trees growth as rods and allow the initiation of crown formation in the spring of 2011.

In the first year, cuts have been carried out in dormant and vegetative phase. The rods were cut in spring to stimulate the emergence of the shoots from viable and well developed buds positioned at the height of $90-100 \mathrm{~cm}$ from the ground. If tree branches formed weak rods in crowning area, they shorten the stump of 2-3 cm, and all axes shorten the height of $90-100 \mathrm{~cm}$ (Fig.1).

In the situation of one year old trees form branches or two years trees with lateral branches, 3-4 ramifications are selected in order to form the first level of the crown and one branch as the central leader. The branches in the first level must have an angle larger than 45 degrees, spaced evenly around the shaft. Branch extending the central axis is shortened after if it exceeds the length of 80-90 $\mathrm{cm}$, and the branches chosen are shortened to $1 / 4$ ? of their length respecting the subordination rule. Short branches with horizontal position above the first floor remain intact. Branches are cut on the trunk at a height of 60-70 cm (Fig.2).

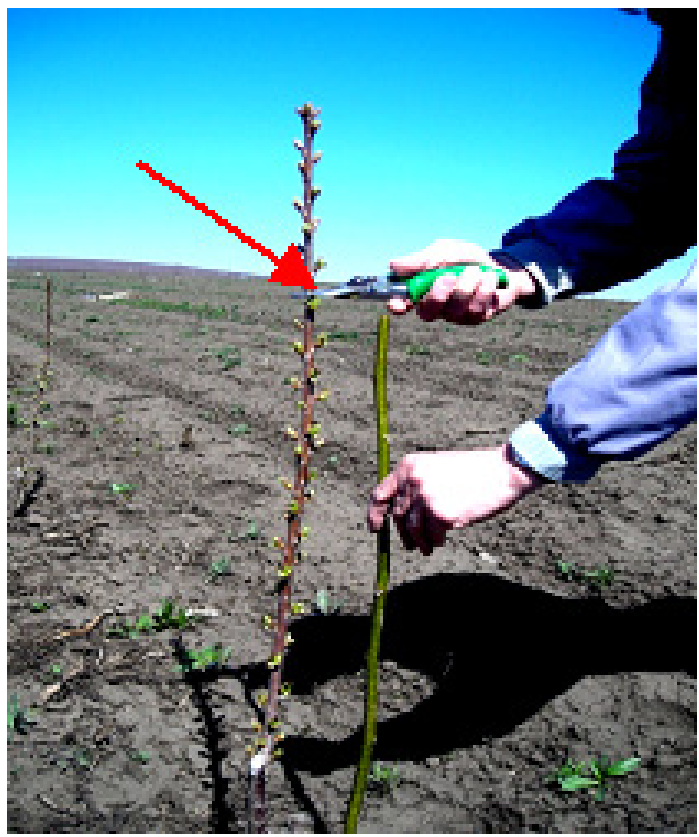

Fig. 1. Shortening rods to $90-100 \mathrm{~cm}$ height.
If the cherry rods are underdeveloped, the graft is shortened to a height of $25-30 \mathrm{~cm}$ from the ground, over one well-developed bud. At the start of vegetation it is allowed to grow one sprig from the respective bud, the rest of the buds being excluded (Fig.3). In June, on the growing shoots appears anticipate shoots which maturation becomes wooded of order I.

After buds burst, on the trees without ramifications, buds are eliminated from the trunk till at 60-70 cm height (Fig.4), 2 well developed buds are chosen at the top but the next 4-5 buds are also ejected (Fig.5). This operation favors the growth of shoot extension of the central axis, avoids natural setting and enables branches with large angle branching.

In spring, when the shoots reach 20-30 $\mathrm{cm}$ length, three of the well-developed lateral ramifications are chosen, according to the requirements mentioned above and one vertical, which will be used as the central axis, its competitors are suppressed, and the other are pinched (Fig. 6). In the case that both terminal buds start growing together generating competing shoots, the weakest shoot is selected for shaft extension and the second one is excluded. The axis stimulates growth at the periphery of the crown and side branches allowing reduced insertion angle to the axis of the scaffold tree. During the growing period of shoots, the sharp angles from

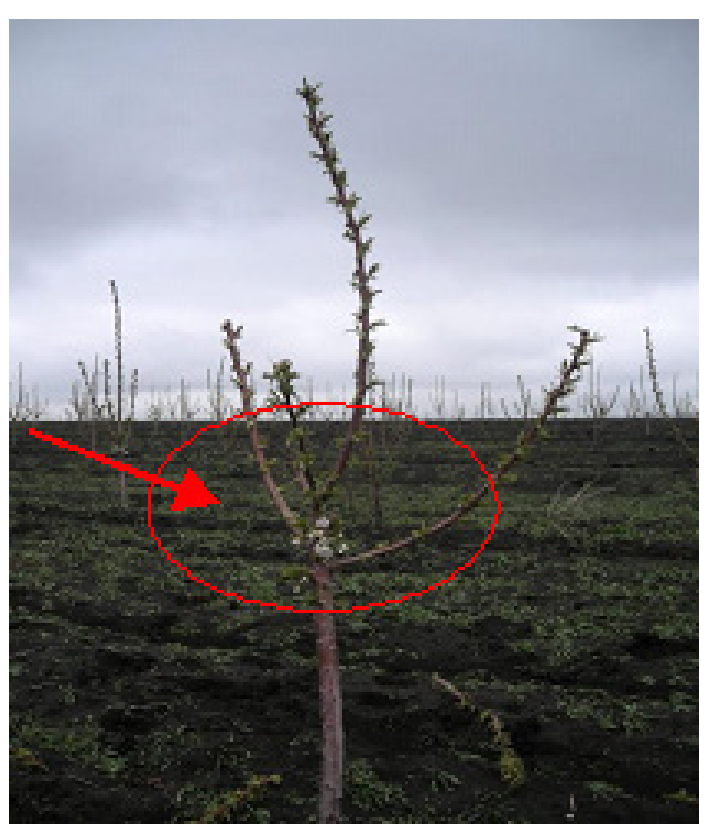

Fig. 2. Projection of first level. 
60 degrees to the vertical are corrected, to form the strong structure and around the same size. The shoots which have an angle greater than the desired insertion angle value are lifted in order to obtain the branches of the same vigor. Trellising branches is done using laundry clips, toothpicks, or links at angles of 60 degrees from the vertical (Fig. 6, 7, 8).

In June, if shoots growths more than $80-90 \mathrm{~cm}$ in length, they are shortened in order to generate

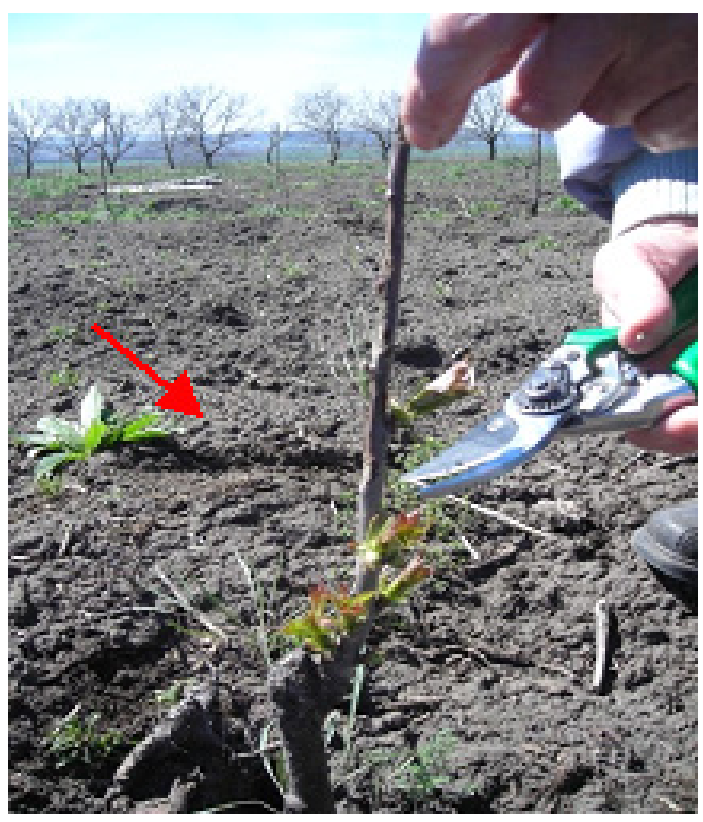

Fig. 3. Cutting rods over a well-developed bud.

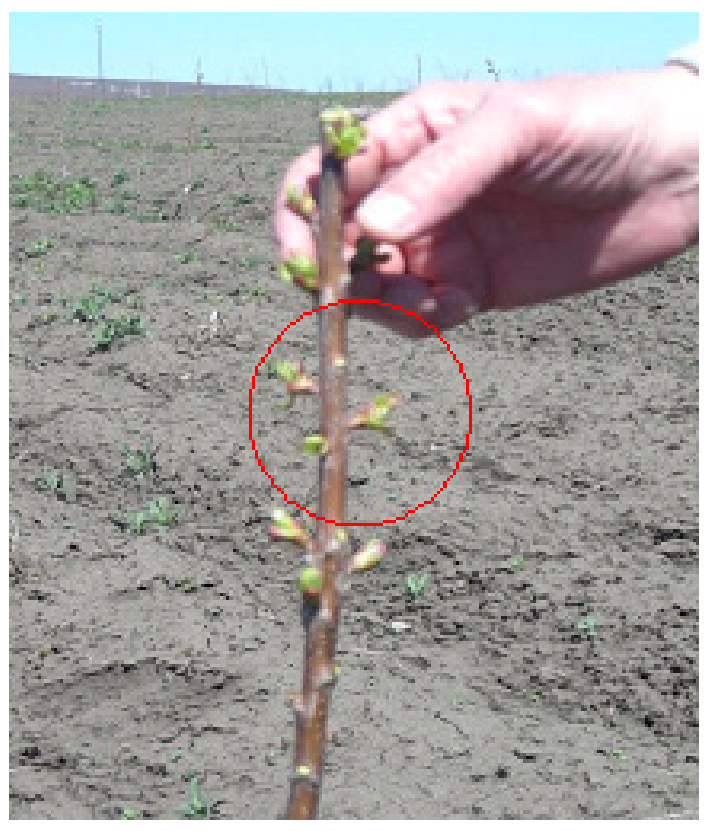

new branches during the growing season of the same year and therefore to decrease the crown formation time. When the young trees form less than 3 shoots, especially in vertical increments trees, when shoots reach a length of 20-30 cm, 15$25 \mathrm{~cm}$ are shortens to cause growth that sprouts early in the summer as mentioned above, is selected for future frameworks and their structure (Fig.9).

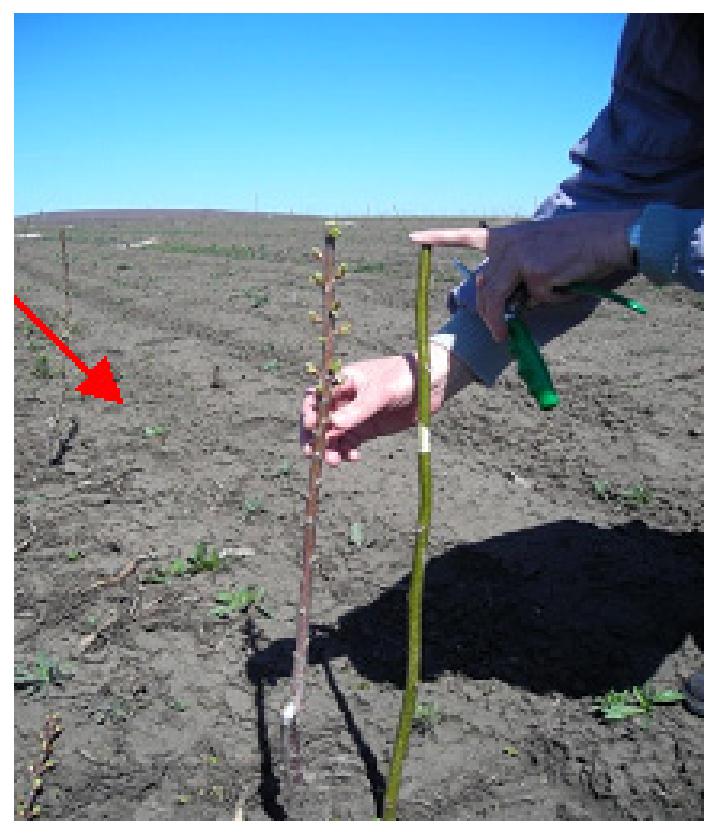

Fig. 4. Blindness buds on the trunk .

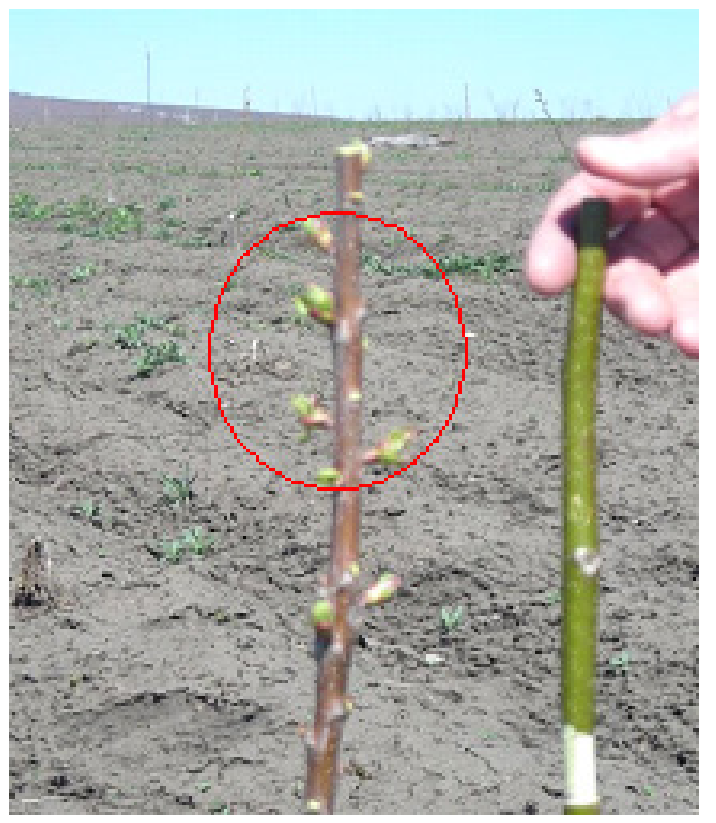

Fig. 5. Blindness 4-5 buds to increase the angle of insertion of branches. 
In the second year, in spring, the chosen structures are shortened to $80-90 \mathrm{~cm}$ in inner bud, unless this was done last summer. Inner bud develops into a vigorous shoot and bud located outside below evolves into a shoot horizontally oriented to the periphery of the crown, which will become an extending branch. The following year, the vigorous vertical branch is eliminated from

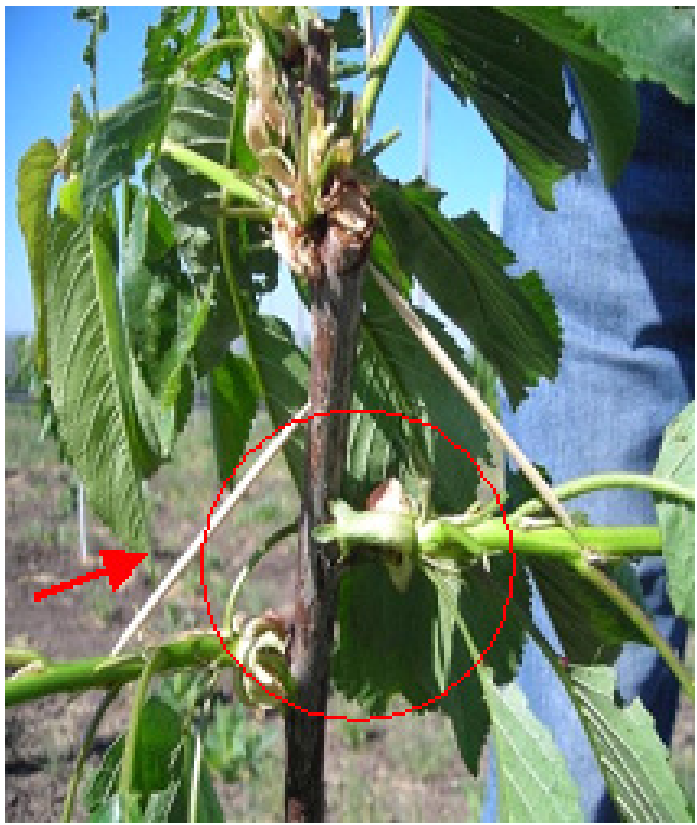

Fig. 6. Installing toothpicks to increase the angle of insertion.

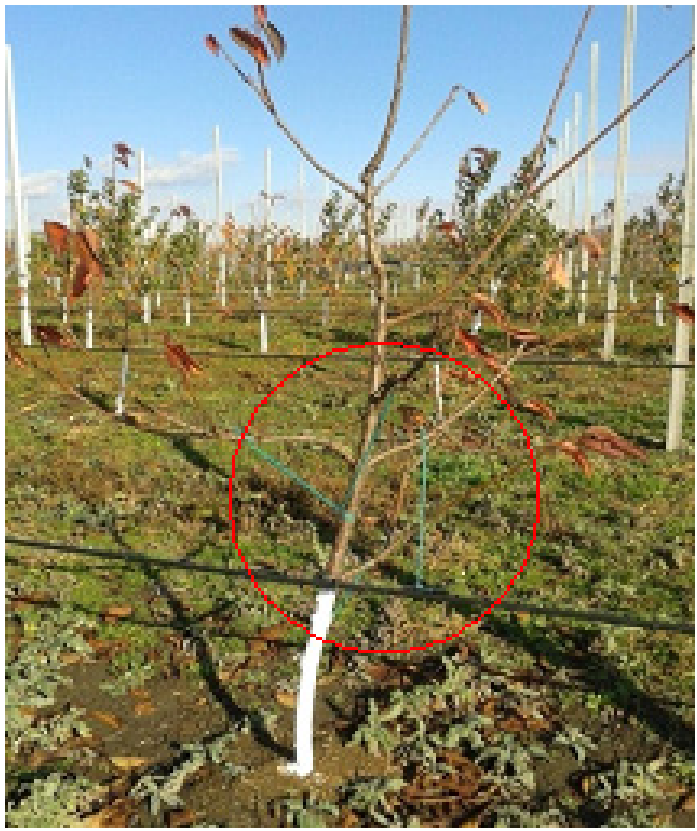

Fig. 8. Trellising branches using links to increase the angle of insertion. ring (Balan 1996) or shortens the stump of 10-15 $\mathrm{cm}$ (Balan et al. 2012) and then transferred to a growth oriented toward horizontal branch (Fig.10, 11).

The sweet cherry is characterized by a strong apical dominance and natural branch setting. To obtain lateral branches in the right positions, it is necessary the grower's intervention. Shortening

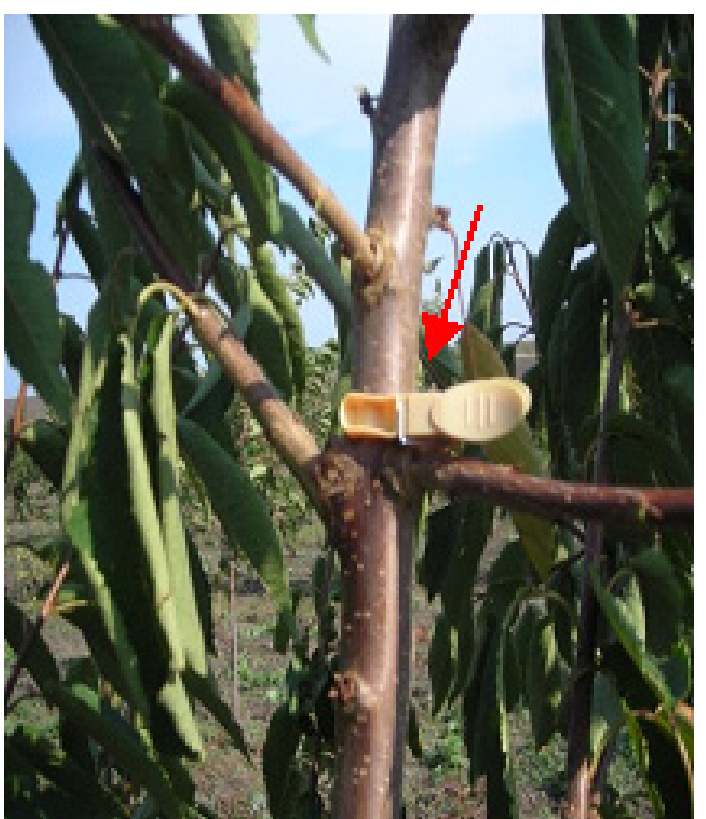

Fig. 7. Installing laundry tongs to increase the angle of insertion.

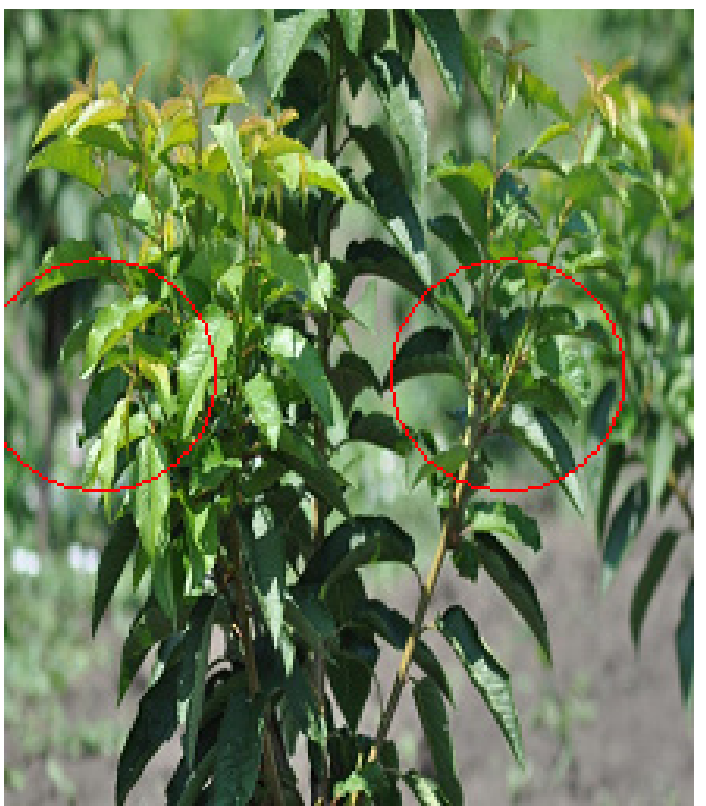

Fig. 9. Anticipating shoots growing. 
annual branches is one of the basic methods to overcome the apical dominance by removing the source of auxins from the top of the branches that inhibit the growth of lateral buds and stimulate lateral branching, just below the cut zone. Branch extension of the central axis is cut to $20-30 \mathrm{~cm}$ above level of shortening of the scaffold branches. Unused branches scaffold formation is suppressed

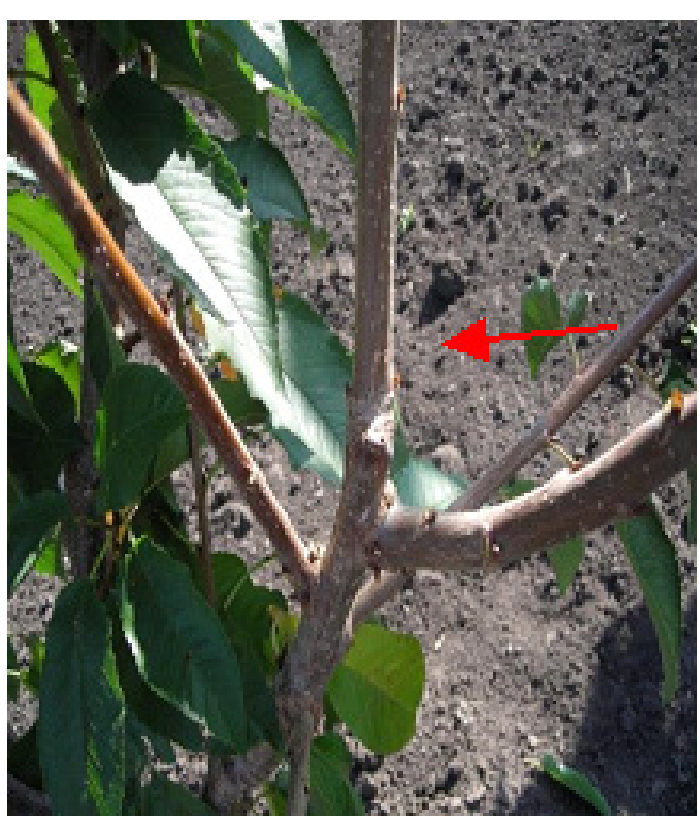

Fig. 10. Inner bud develops into a vigorous shoot.

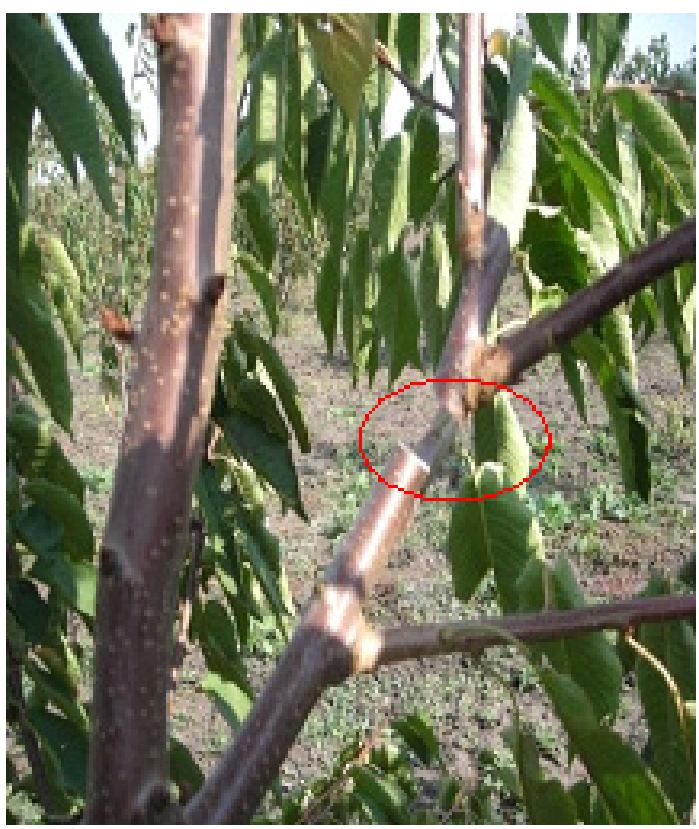

Fig.12.Using incisions to induce branching. or shortened at the stump of $10-15 \mathrm{~cm}$. The frameworks are shortened to get closer to the axis of the tree branches to design secondary branches at a distance of $30-40 \mathrm{~cm}$ from the shaft and from each other, and branch extension shaft - to form the second level branches snake. To get the branches on the ax in necessary places, incisions are performed above the bud, cutting liberian

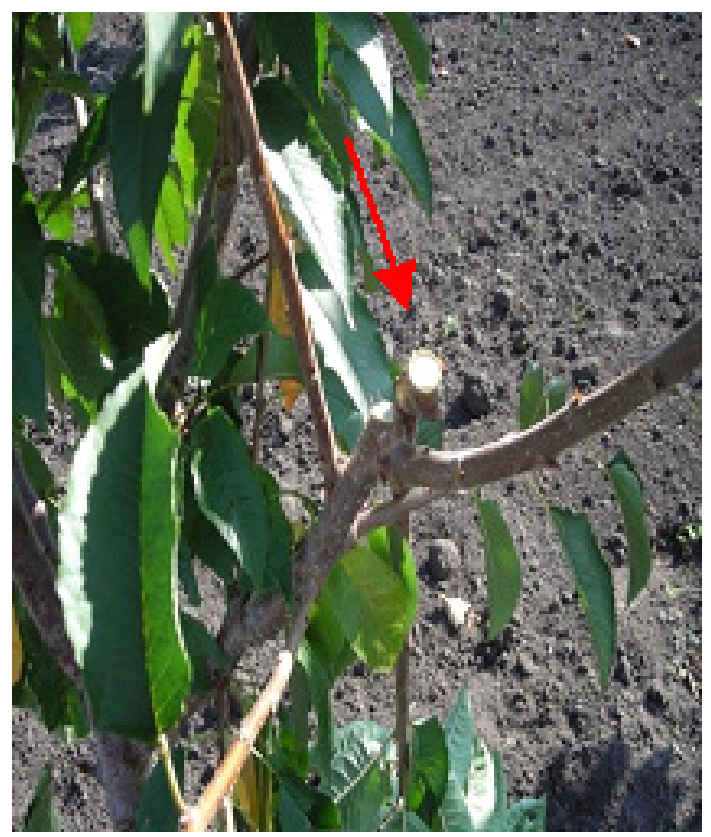

Fig. 11. Outer bud develops into a shoot horizontally oriented.

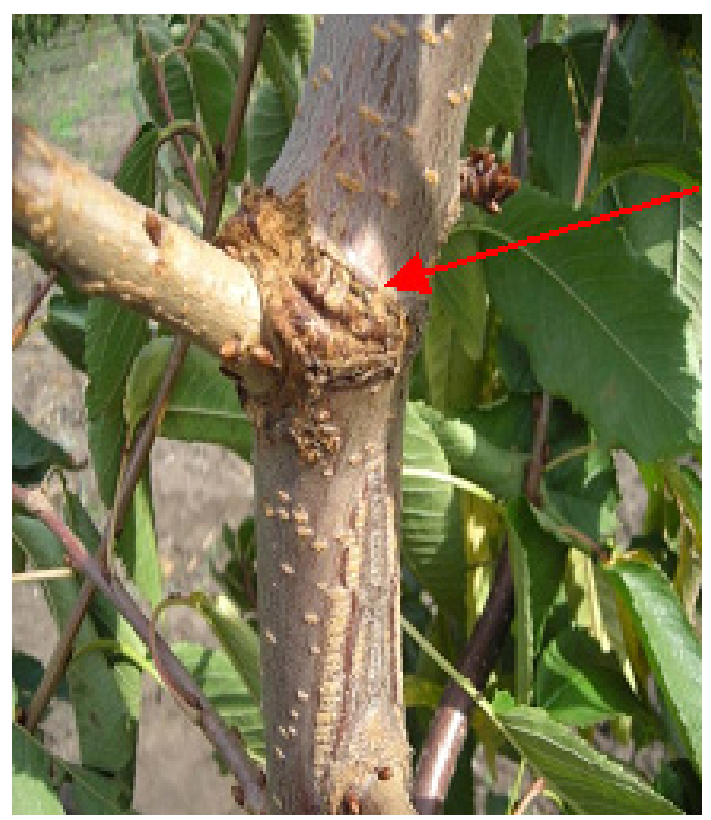

Fig. 13. Cutting to increase branching angle roof structure. 


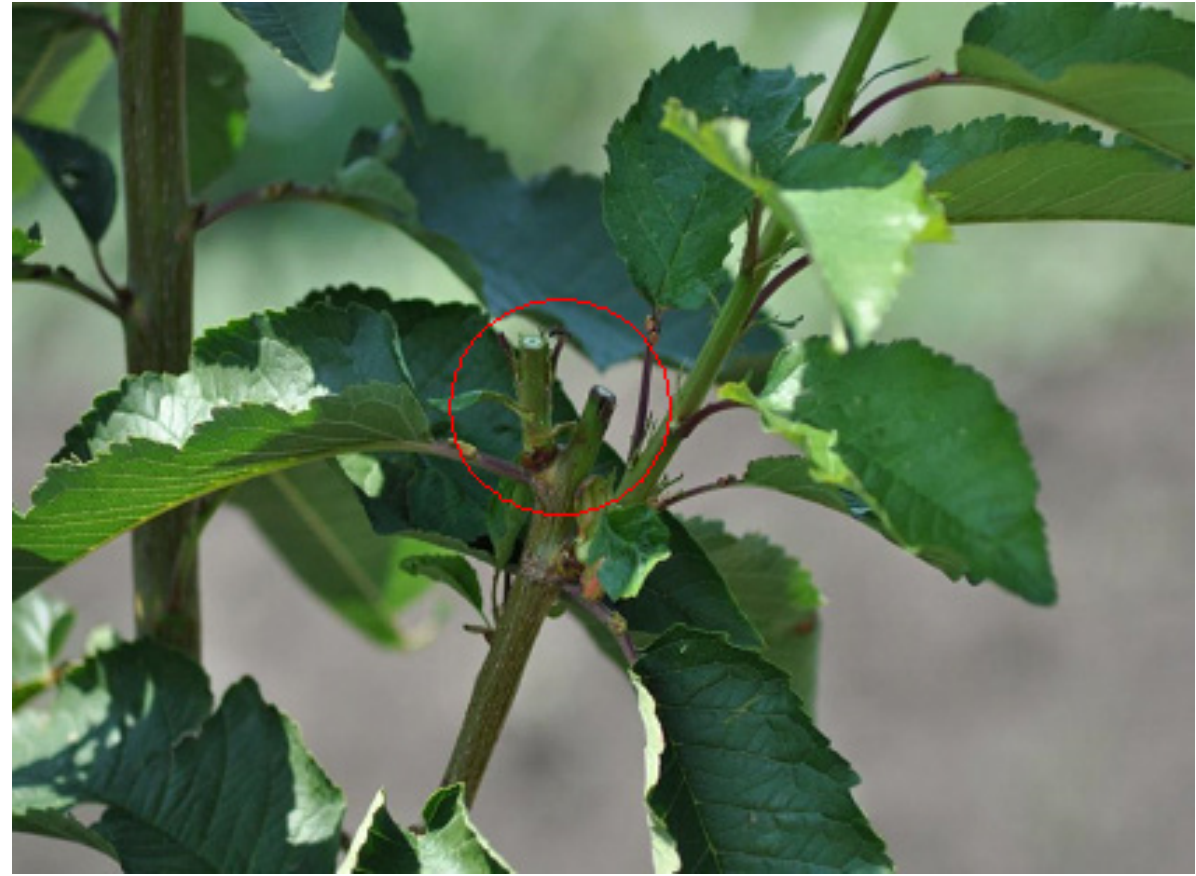

Fig. 14. Cutting the pin vertical shoots.

vessels and 2-3 rings of sapwood (Fig.12). During vegetation period, when the shoots reach a length of 20-30 cm, 3-4 shoots are selected on each branch, one will be used to extend the axe and 2-3 shoots to form the first secondary branches.

The concurring lengthening twigs or the shoots and vertical twigs are weeded; the others are pinched at $15 \mathrm{~cm}$. The branch which will be used to extend the branches will be marginal and not at an angle less than 60 degrees from vertical. Twigs used for the formation of the secondary branches will need to form an angle greater than 60 degrees also from vertical.

In the third year, in spring, branches extending the central axis are shortened to 80$90 \mathrm{~cm}$ scaffold to cause the appearance of new shoots for branches and secondary branches. The branches chosen for the second level of branches with an angle of insertion larger than previous ones are balanced in growth vigor. To increase the angle of branching the above cutting method is used (Fig.13).

On each of the branches on the first level, secondary branches ranches are projected. The branches chosen for secondary branches with angle greater than 60 degrees should not be shortened, to begin to form fruit buds and speed up the entrance of trees in production. On the branches and the secondary branches the thick branches that exceed half the vigor that grow vertically oriented and stump are cut, following Zahn's method, cited by Cimpoieş 2012 (Fig.14).

During vegetation, in the case that vigorous growth is formed in the crown, more than 80-90 $\mathrm{cm}$, until mid-June, it can be pinched their peak during this period, to branch and rush trees to fruit.

To achieve high fruit quality in intensive orchards and cherry it is necessary to have annual branches of different lengths. The number of flowering buds on the branches of their length varies yearly (Tab. 1). The 'Skeena' cultivar has short branches up to $20 \mathrm{~cm}$ long, the inflorescences $26.9 \%$ are placed on the branches of $20-40 \mathrm{~cm}$ length $-43.6 \%$ and $40-80 \mathrm{~cm}$ long branches $-29.5 \%$. The same regularity is recorded in 'Adriana' and 'Ferrovia' cultivars. Therefore, during growth and fructification of sweet cherry trees, it is necessary to bear in crown young branches, 1 to 4 years old, with high biological potential and with an average length of $30-45 \mathrm{~cm}$ at least, and at a back to $4-5$ leaves harvested fruit.

The density of the cherry bouquets depends not only on the length of the branches but also on their age (Tab.2). In the case of 3 years old wood, 'Ferrovia' showed the highest density of 50.2 bouquets more pcs / linear meter and 'Adriana' 
Tab. 1. The number of flowering shoots at the annual cherry branches of different lengths, pcs

\begin{tabular}{|c|c|c|c|c|c|c|c|}
\hline \multirow{2}{*}{ Cultivar } & \multirow{2}{*}{$\begin{array}{l}\text { Number of } \\
\text { flowering } \\
\text { shoots }\end{array}$} & \multicolumn{6}{|c|}{ Length of branches } \\
\hline & & $0-20 \mathrm{~cm}$ & $\%$ & $20-40 \mathrm{~cm}$ & $\%$ & $40-80 \mathrm{~cm}$ & $\%$ \\
\hline Skeena & 101.6 & 27.3 & 26.9 & 44.3 & 43.6 & 30.0 & 29.5 \\
\hline Adriana & 86.2 & 24.3 & 28.2 & 36.6 & 42.5 & 25.3 & 29.3 \\
\hline Ferrovia & 102.0 & 28.0 & 27.4 & 44.0 & 43.1 & 30.0 & 29.5 \\
\hline Average & 96.6 & 31.9 & 33.0 & 36.3 & 37.6 & 28.4 & 29.4 \\
\hline
\end{tabular}

Tab. 2. Density of cherry wood bouquets at 2 and 3 years old.

\begin{tabular}{ccccccc}
\hline & \multicolumn{3}{c}{ Wood at 3 years } & \multicolumn{3}{c}{ Wood at 2 years } \\
\cline { 2 - 7 } Cultivar & Length, cm & $\begin{array}{c}\text { Bouquets, } \\
\text { pieces }\end{array}$ & $\begin{array}{c}\text { Bouquets, } \\
\text { pieces/m }\end{array}$ & Length, cm & $\begin{array}{c}\text { Bouquets, } \\
\text { pieces }\end{array}$ & $\begin{array}{c}\text { Bouquets, } \\
\text { pieces/m }\end{array}$ \\
\hline Skeena & 34.3 & 9.0 & 26.2 & 23.7 & 7.3 & 30.8 \\
\hline Adriana & 30.8 & 9.8 & 25.8 & 21.1 & 7.3 & 34.5 \\
\hline Ferrovia & 31.1 & 15.6 & 50.2 & 21.3 & 9.8 & 46.1 \\
\hline Average & 32.1 & 11.5 & 35.8 & 22.0 & 8.1 & 36.8 \\
\hline
\end{tabular}

showed the lowest density (25.8 pieces/linear meter).

In the case of the wood at two years the bud density is also higher for 'Ferrovia' and is 46,1 bouquets more per linear meter of branch. From the data presented it cannot be said with certainty that the cherry cultivars 'Skeena', 'Adriana' and 'Ferrovia' will get the same results in the period of full production, but certainly we can say that the branches of fruit placed on wood of 2-3 years old are very productive.

In the fourth year and the following years the work of the previous years is repeated on new extensions of annual branches. Definitive branches are chosen for the second level, which balance each other and branch extension shaft is shortened to $25-30 \mathrm{~cm}$ above the level of the cutting of level branches. On the first layer structures and semi skeletal branches are formed. Vigorous branches, located on the shaft between layers, shorten the stump of $10-15 \mathrm{~cm}$, in order to be further transformed by cutting the branches. The angle of inclination of the scaffold is corrected by the cutting described above, higher order subordinate branches of the inferior. The morphology of cherry tree is influenced by biological of the cultivar (Tab.3).

Sum length of branches of 'Adriana', 'Skeena' and 'Ferrovia', in the $5^{\text {th }}$ year of vegetation is from 50.7 to $56.2 \mathrm{~m} /$ tree, including the annual branches - more than $80 \%$. The number of annual branches, in the first two years of vegetation in the orchard, grows moderately, but in the next two years shows geometric progression, increasing in year five of vegetation to 64.3 to $72.3 \mathrm{pcs} /$ tree.

The average length of annual branches is about $65 \mathrm{~cm}$ and a base number in the process of crown formation. The best length of annual increases to hasten their entry formation of fruit trees is $80-90 \mathrm{~cm}$. The number of bouquets varies and is directly proportion to the length and age of the branches. Therefore, intense growth of trees in the early years of vegetation allows rapid formation of crown design parameters.

\section{CONCLUSION}

Formation of cherry trees after natural system improved with low volume combines with the use of small and medium rootstocks extends vertically to $2.5-3 \mathrm{~m}$ tall. The trees were led by the crown trunk 70-80 $\mathrm{cm}$ high and a shaft well developed, 
Tab. 3. Morphology of cherry trees after training system „Improved natural crown with low volume" (5 years old tree, 2014)

\begin{tabular}{ccccc}
\hline $\begin{array}{c}\text { Age of branches, } \\
\text { years }\end{array}$ & $\begin{array}{c}\text { Sum length of } \\
\text { branches, } \mathrm{m}\end{array}$ & $\begin{array}{c}\text { Number of branches, } \\
\text { pieces }\end{array}$ & $\begin{array}{c}\text { Average length of } \\
\text { brances, cm }\end{array}$ & $\begin{array}{c}\text { Bouquets, } \\
\text { pieces. }\end{array}$ \\
\hline 1 & \multicolumn{5}{c}{ cv 'Adriana' } & 67.2 & - \\
\hline 2 & 43.3 & 64.3 & 57.4 & 139 \\
\hline 3 & 5.9 & 10.3 & 41.2 & 46.3 \\
\hline 4 & 2.4 & 5.6 & 36.9 & 6.6 \\
\hline Total & 1.2 & 3.3 & - & - \\
\hline & 52.8 & 83.5 & 64.1 & - \\
\hline 1 & & cv 'Skeena' & 132 \\
\hline 2 & 46.3 & 72.3 & 56.8 & 79 \\
\hline 3 & 5.8 & 10.3 & 50.9 & - \\
\hline 4 & 3.0 & 6 & 31.3 & - \\
\hline Total & 1.1 & 6 & - & 119 \\
\hline & 56.2 & 94.6 & 64.1 & 54.6 \\
\hline 1 & & 65.3 & 49.0 & - \\
\hline 2 & 42.2 & 12 & 42.4 & \\
\hline 3 & 5.8 & 6 & 36.9 & - \\
\hline 4 & 2.5 & 3.3 & & \\
\hline Total & 1.2 & 86.6 & & \\
\hline
\end{tabular}

which have inserted 2 levels of braches at intervals of $80-90 \mathrm{~cm}$. In the first level, 3 to 4 branches are arranged uniformly around the axis in spiral at a distance of 8-12 cm one from each other; threesections extend at a height of $80-90 \mathrm{~cm}$ from the top or from $150-180 \mathrm{~cm}$ from the ground, and in third level - only garnishing branches. All structures of the extension must be the angle from vertical of more than 60 degrees and the opening angle forming between them, from the horizontal plane, 90-120 degrees. The frameworks of the second layer should extend in space the structures of the first level. On every branches structure are located 2-3 secondary branches bilateral-altern distributed at a distance of $30-40 \mathrm{~cm}$ from the axis and each other. On axe and the basic branches fruiting formations is garnishing the branches.

\section{REFERENCES}

1. Asanică A, Petre Gh, Petre V(2013). The establishment and operation of cherry ochards and cherry ,Cireș.

2. Babuc V (2012). Pomiculture,Tipografia Centrală .

3. Balan V, Cimpoieş Gh, Barbaroş M (2001). Pomiculture, Museum .

4. Balan V (2009). Culture system in horticulture. The yield fruit production, Academos.

5. BalanV (2012). Cherry crops prospects. Pomiculture, viticulture and winemaking in Moldova.

6. Balan V, Peșteanu A, Ivanov I, Vămăşescu S, Tîrsina 0 (2012). The process of cutting the branches of apple tree. Patent.MD 537 Z 2013.03.31. BOPI nr. 8/2012

7. Balan V. The process of cutting the branches of apple tree. Patent. RM, nr. 360. Publ.: BOPI nr. 1/96, 4 p.

8. Cimpoieș Gh (2001). Special pomiculture.

9. Ghena N, Braniște N, Stănică F (2004). General pomiculture, Matrix Rom .

10. Long LE, Long M, Peşteanu A, Gudumac E (2014). Cherries production. Tehnological manual. 